\title{
Duluth at SemEval-2019 Task 6: Lexical Approaches to Identify and Categorize Offensive Tweets
}

\author{
Ted Pedersen \\ Department of Computer Science \\ University of Minnesota \\ Duluth, MN 55812 USA \\ tpederseed. umn. edu
}

\begin{abstract}
This paper describes the Duluth systems that participated in SemEval-2019 Task 6, Identifying and Categorizing Offensive Language in Social Media (OffensEval). For the most part these systems took traditional Machine Learning approaches that built classifiers from lexical features found in manually labeled training data. However, our most successful system for classifying a tweet as offensive (or not) was a rule-based black-list approach, and we also experimented with combining the training data from two different but related SemEval tasks. Our best systems in each of the three OffensEval tasks placed in the middle of the comparative evaluation, ranking $57^{\text {th }}$ of 103 in task A, $39^{\text {th }}$ of 75 in task B, and $44^{\text {th }}$ of 65 in task C.
\end{abstract}

\section{Introduction}

Social media is notorious for providing a platform for offensive, toxic, and hateful speech. There is a pressing need for NLP tools that can identify and moderate this type of content.

The OffensEval task (Zampieri et al., 2019b) focuses on identifying offensive language in tweets, and determining if specific individuals or groups are being targeted. Our approach was to rely on traditional Machine Learning methods as implemented by Scikit (Pedregosa et al., 2011) to build classifiers from manually labeled examples of offensive tweets. Our models relied on lexical features, including character ngrams, words, and word ngrams. We also included a dictionary based black-list approach, and experimented with combining training data from two related yet different SemEval-2019 tasks.

Offensive language is an umbrella term that covers hate speech, cyber-bullying, abusive language, profanity, and so on. Recognizing offensive language is an important first step in dealing with different kinds of problematic text. Lan- guage that is offensive may simply violate community standards regarding the use of profanity, but in other cases may cross over to become abusive, threatening, or dangerous. Detecting such language has proven to be a challenging problem, at least in part because it remains difficult to make distinctions between the casual and even friendly use of profanity versus more serious forms of offensive language (Fortuna and Nunes, 2018; Schmidt and Wiegand, 2017).

\section{Experimental Data}

OffensEval is made up of three tasks that were carried out in stages during January 2019. Task $\mathrm{A}$ is to classify a tweet as being offensive (OFF) or not (NOT). Task B takes the offensive tweets from task A and decides if they are targeted insults (TIN) or not (UNT). Task C looks at the targeted insults from task B and classifies them as being directed against an individual (IND), group (GRP) or other entity (OTH).

Task A provides 13,240 training tweets, of which $8,840(66.7 \%)$ were not offensive (NOT). Task B is made up of the 4,400 training tweets that were offensive (OFF), where 3,876 (88.1\%) of these are targeted insults (TIN). Task C includes the targeted insults from task B, of which 2,407 $(62.1 \%)$ were targeted against individuals (IND) and 1,074 (27.7\%) were against groups (GRP). Additional details about the task data can be found in (Zampieri et al., 2019a)

The distribution of classes in the evaluation data was similar. Task A has 860 evaluation tweets of which $620(72 \%)$ were not offensive. Task B includes 240 offensive evaluation tweets, where 213 (89\%) were targeted insults. These made up the evaluation data for task C, where $100(47 \%)$ were against individuals, and $78(37 \%)$ were against groups. 
The amount of training data is modest, particularly for tasks B and C. In addition, the classes in Task B are quite skewed. Given these factors we decided to rely on traditional Machine Learning techniques, since these have the potential to perform well even with limited training data.

\section{System Descriptions}

We created three systems for each of the three tasks. Preprocessing was very simple : tweets were converted to lower case and then tokenized into character ngrams, words, or word ngrams.

In task A we relied on unigram models. However, for tasks $\mathrm{B}$ and $\mathrm{C}$ we focused on character ngrams in order to try and find regularities in the smaller amounts of training data available for these tasks.

\subsection{Task A}

The goal of Task A was to classify a tweet as offensive (OFF) or not (NOT). We relied on the default settings for vectorization and Machine Learning as provided in Scikit, except as noted below. This results in tokenization based on space separated strings where punctuation and other nonalphanumeric characters are discarded.

A-Sub1 is a Logistic Regression classifier that weighs unigram features using tf-idf.

A-Sub2 is the same as A-Sub1 except that the training data is augmented with the training data from Semeval-2019 Task 5 HatEval (Basile et al., 2019), a task that identifies hate speech. It provides 22,240 training examples where 14,057 $(65.2 \%)$ are not hate speech. We made the obviously incorrect assumption that tweets that aren't hate speech would also not be offensive. We had hoped that doubling the amount of training data would improve our performance despite our flawed assumption (although it did not).

A-Sub3 is a very simple rule based on a blacklist created by merging the following sources:

- words or terms used in offensive tweets five or more times in the OffensEval training data,

- words or terms used in hateful tweets five or more times in the HatEval training data, and

- black-lists found via web search said to be used by WordPress, Google, Facebook, and Youtube $^{1}$.

\footnotetext{
${ }^{1}$ https://www.freewebheaders.com/
}

Any word or term that appears in two or more of these lists is selected, leading to a master blacklist of 563 words. Any tweet that includes any of these words is labeled as offensive (OFF).

\subsection{Task B}

Task B seeks to identify if an offensive tweet is a targeted insult (TIN) or not (UNT). All three systems relied on character-based ngrams and the default Scikit settings for vectorization and the specific learning method.

B-Sub1 learns a random forest classifier, and BSub2 learns a decision tree. Both represent features as 3 alphanumeric character sequences.

B-Sub3 learns a linear Support Vector Machine from the training data. Features are represented as 3 non-space character sequences. We used nonspace characters in this case in order to capture special characters that would be discarded by BSub1 and B-Sub2. We were particularly interested in retaining \# (hashtags) and @ (user ids).

\subsection{Task C}

All tweets in task C are targeted insults (TIN). The goal is to identify if the target is an individual (IND), group (GRP), or some other entity (OTH).

All of these systems used the default settings from Scikit for vectorization and Machine Learning, except as noted below.

C-Sub1 learns a multinomial Naive Bayesian classifier from the training data. Features are simple unigrams made up of alpa-numeric characters. During development we noticed that Naive Bayes tended to find more balanced distributions of classes than our other approaches.

C-Sub2 learns a decision tree classifier from the training data. Features are 3 character sequences. During development we observed that this was the only method that assigned tweets to the other (OTH) category.

C-Sub3 learns a logistic regression classifier from the training data. Features are word ngrams made up of sequences of 1,2 and 3 words that occur in more than 10 tweets in the training data.

\section{Experimental Results}

The official rankings in OffensEval were based on macro-averaged F1, and accuracy was also reported. The performance of individual classes was measured by Precision, Recall, and the $F 1$ score. 


\begin{tabular}{lcc} 
System & F1-Macro & Accuracy \\
\hline A-TOP & .83 & \\
A-Sub3 & .73 & .80 \\
A-Sub2 & .69 & .80 \\
A-Sub1 & .68 & .80 \\
A-Baseline & .42 & .72 \\
\hline B-TOP & .76 & \\
B-Sub1 & .60 & .88 \\
B-Sub2 & .57 & .82 \\
B-Sub3 & .52 & .77 \\
B-Baseline & .47 & .89 \\
\hline C-TOP & .66 & \\
C-Sub1 & .48 & .67 \\
C-Sub3 & .45 & .62 \\
C-Sub2 & .42 & .53 \\
C-Baseline & .21 & .47
\end{tabular}

Table 1: Duluth OffensEval Results

The results of the Duluth Systems are summarized in Table 1 . X-TOP is the $1^{\text {st }}$ ranked system in each task. X-Baseline assigns each test tweet to the most frequent class in the training data.

Next, we'll examine the results from each task in more detail. In the confusion matrices provided, the distribution of gold answers (ground truth) is shown on the rows, and the system predictions are on the columns.

\subsection{Task A}

Task A asks whether a tweet is offensive (or not). It had the largest amount of training data $(13,240$ examples), of which $33 \%$ were considered offensive (OFF) and 67\% were not (NOT). In Table 2 and the discussion that follows a true positive is a tweet that is known by ground truth to be not offensive (NOT) and that is predicted to be not offensive (NOT). A true negative is a tweet that is known to be offensive (OFF) and is predicted to be offensive (OFF).

\subsection{Confusion Matrix Analysis}

A-Sub3 had a modest advantage over the other two systems. A-Sub3 was a simple rule-based blacklist approach, while A-Sub1 and A-Sub2 used Machine Learning. All three systems scored identical accuracy $(80 \%)$, but in looking at their confusion matrices some interesting differences emerge.

Table 2 shows that the rule based method ASub3 has a much smaller number of false negatives (112 versus 160 and 152). It also has a larger

\begin{tabular}{lrrr|rrr}
\hline & NOT & OFF & & P & R & F1 \\
NOT & 609 & 11 & 620 & .79 & .98 & .88 \\
OFF & 160 & 80 & 240 & .88 & .33 & .48 \\
& 769 & 91 & 860 & .82 & .80 & .77
\end{tabular}

A-Sub 1: Logistic Regression

\begin{tabular}{lrrr|rrr}
\hline & NOT & OFF & & $\mathrm{P}$ & $\mathrm{R}$ & $\mathrm{F} 1$ \\
NOT & 602 & 18 & 620 & .80 & .97 & .88 \\
OFF & 152 & 88 & 240 & .83 & .37 & .51 \\
& 754 & 106 & 860 & .81 & .80 & .77
\end{tabular}

A-Sub 2: Logistic Reg + HatEval

\begin{tabular}{rrrr|rrr}
\hline & NOT & OFF & & P & R & F1 \\
NOT & 558 & 62 & 620 & .83 & .90 & .87 \\
OFF & 112 & 128 & 240 & .67 & .53 & .60 \\
& 670 & 190 & 860 & .79 & .80 & .79
\end{tabular}

A-Sub3 : Rule Based, black-list

Table 2: Task A Confusion Matrices

number of true negatives (128 versus 80 and 88 ). Overall the rule based system finds more tweets offensive (190) than the Machine Learning methods (91 and 106). This happens because our rule based system only needs to find a single occurrence of one of our 563 black-listed terms to consider a tweet offensive, no doubt leading to many non-offensive tweets being considered offensive (62 versus 11 and 18).

The only difference between A-Sub1 and ASub2 was that A-Sub2 had approximately double the number of training tweets. The extra tweets were from the SemEval-2019 hate speech task (HatEval). We hypothesized that more training data might help improve the results of a logistic regression classifier (which was used for both ASub1 and A-Sub2). After increasing the training data, A-Sub2 is able to classify exactly one more tweet correctly (690 versus 689 ). We were somewhat surprised at this very limited effect, although the HateEval corpus is focused on a particular domain of hate speech where the targets are women and immigrants. This does not appear to have matched well with the OffensEval training data. 


\begin{tabular}{l|l} 
NOT offensive & \multicolumn{1}{c}{ OFFensive } \\
\hline user & SHIT \\
antifa & FUCK \\
url & BITCH \\
best & STUPID \\
thank & ASS \\
conservatives & FUCKING \\
new & IDIOT \\
beautiful & LIAR \\
here & DISGUSTING \\
brexit & SUCKS \\
thanks & SICK \\
love & CRAP \\
she & RACIST \\
day & DUMB \\
awesome & FASCIST \\
adorable & NIGGA \\
safe & FUCKED \\
voting & CRAZY \\
funny & IGNORANT \\
stand & FOOL \\
justice & COWARD \\
idea & IDIOTS \\
there & SUCK \\
right & KILL \\
join & PUSSY \\
well & UGLY \\
amazing & WORST \\
twitter & DAMN \\
welcome & BULLSHIT \\
trying & ASSHOLE \\
& \\
\hline
\end{tabular}

Table 3: Task A Feature Analysis - A-Sub1

\begin{tabular}{l|l} 
NOT offensive & \multicolumn{1}{c}{ OFFensive } \\
\hline https & BITCH \\
co & BUILDTHATWALL \\
immigrant & SHIT \\
men & WOMENSUCK \\
antifa & FUCK \\
url & ASS \\
user & ILLEGAL \\
ram & BITCHES \\
thank & SUCKS \\
best & NODACA \\
new & BUILDTHEWALL \\
conservatives & STUPID \\
when & LIAR \\
son & IDIOT \\
you & DISGUSTING \\
stand & WHORE \\
beautiful & FUCKING \\
kunt & HOE \\
love & SUCK \\
justice & ILLEGALS \\
facebook & SICK \\
ho & FASCIST \\
tonight & CRAP \\
thanks & IGNORANT \\
wondering & THESE \\
day & RACIST \\
accused & KILL \\
brexit & CRAZY \\
alone & DUMB \\
twitter & WHITE \\
& \\
\hline
\end{tabular}

Table 4: Task A Feature Analysis - A-Sub2

In Tables 3 and 4 we bold face the 18 features that were shared between A-Sub1 and ASub2. This gives us some insight into the impact of merging the OffensEval and HatEval training data. Some generic offensive features remain in Table 4 but are strongly augmented by HatEval features that are oriented against women and immigrants.

The 13 shared terms that were indicative of the not offensive class are shown in italics. Some features are what we'd expect for non-offensive tweets : love, beautiful, thanks, thank, justice and best. Others are more artifacts of the data, user is an anonymized twitter id and $u r l$ is an anonymized web site. Others are less clearly not offensive, and seem related to political conversation : antifa, conservatives, and brexit. 


\begin{tabular}{rlrl} 
Ratio & Feature & OFF & NOT \\
\hline 61.0 & BITCH & 61 & 0 \\
17.5 & IDIOT & 35 & 2 \\
14.0 & ASSHOLE & 14 & 0 \\
10.6 & FUCK & 106 & 10 \\
10.2 & STUPID & 92 & 9 \\
10.0 & DICK & 10 & 1 \\
10.0 & BITCHES & 10 & 0 \\
9.0 & SHIT & 278 & 31 \\
9.0 & RAPIST & 9 & 1 \\
7.3 & FUCKED & 22 & 3 \\
6.3 & FUCKING & 82 & 13 \\
5.8 & SUCKS & 35 & 6 \\
5.5 & CRAP & 33 & 6 \\
5.3 & IDIOTS & 16 & 3 \\
5.0 & SCUM & 10 & 2 \\
5.0 & MORON & 10 & 2 \\
4.9 & ASS & 108 & 22 \\
4.8 & IGNORANT & 19 & 4 \\
4.5 & LOSER & 9 & 2 \\
4.3 & SHITTY & 13 & 3 \\
4.2 & BUTT & 17 & 4 \\
4.0 & UGLY & 12 & 3 \\
3.8 & DUMB & 23 & 6 \\
3.2 & PUSSY & 13 & 4 \\
3.2 & NIGGA & 16 & 5 \\
3.0 & PORN & 9 & 3 \\
2.9 & HELL & 38 & 13 \\
2.9 & BULLSHIT & 23 & 8 \\
2.6 & SUCK & 21 & 8 \\
2.5 & KILL & 32 & 13
\end{tabular}

Table 5: Task A Feature Analysis - A-Sub3

However, there are some inconsistencies to note. In Table 3 NIGGA is not necessarily an offensive term and points to the need for annotators to have subtle understandings of culture (Waseem et al., 2018). In Table 4 kunt is a deliberate misspelling meant to disguise intent (c.f. (Gröndahl et al., 2018)).

Table 5 shows the top 30 terms from our blacklist system A-Sub3 that proved to be most discriminating in identifying an offensive tweet. Recall that A-Sub3 had the highest F1-Macro score of our task A systems. The first column shows a simple ratio of the number of times a feature is used in an offensive tweet (OFF in $3^{\text {rd }}$ column) versus a not offensive one (NOT in $4^{\text {th }}$ column). The most discriminating feature BITCH occurred in 61 offensive tweets and in 0 that were not offensive.

\begin{tabular}{lrrr|rrr}
\hline & TIN & UNT & & P & R & F1 \\
TIN & 206 & 7 & 213 & .90 & .97 & .93 \\
UNT & 22 & 5 & 27 & .42 & .19 & .26 \\
& 228 & 12 & 240 & .85 & .88 & .86
\end{tabular}

B-Sub1 : Random Forest

\begin{tabular}{lrrr|rrr}
\hline & TIN & UNT & & $\mathrm{P}$ & $\mathrm{R}$ & $\mathrm{F} 1$ \\
TIN & 188 & 25 & 213 & .90 & .88 & .89 \\
UNT & 20 & 7 & 27 & .21 & .26 & .24 \\
& 208 & 732 & 240 & .83 & .81 & .82
\end{tabular}

B-Sub2 : Decision Tree

\begin{tabular}{lrrr|rrr}
\hline & TIN & UNT & & P & R & F1 \\
TIN & 179 & 34 & 213 & .90 & .84 & .87 \\
UNT & 21 & 6 & 27 & .15 & .22 & .18 \\
& 200 & 40 & 240 & .81 & .77 & .79
\end{tabular}

B-Sub3 : Linear SVM

Table 6: Task B Duluth Systems

\subsection{Task B}

Task B includes 4,400 training tweets, all of which are judged by ground truth to be offensive. This is a fairly modest amount of training data, particularly given how noisy tweets tend to be. As a result we shifted from using unigrams as features (as in Task A) and moved to the use of character ngrams, in the hopes of identifying patterns that may not exist at the unigram level.

The data in Task B is also the most skewed of all the tasks. Nearly $90 \%$ of the tweets belonged to the class of targeted insult (TIN). Our three Task B systems used different Machine Learning classifiers, and all tended to produce very skewed results, where most tweets were judged to be targeted insults (TIN). This is clearly illustrated in Table 6, which shows that the random forest classifier (B-Sub1) was better in terms of Precision and Recall for TIN, whereas all three classifiers struggled with the UNT class.

\subsection{Task C}

Task $\mathrm{C}$ had an even smaller amount of training data (3,876 instances). Given a targeted insult, systems were asked to decide if the target an individual (IND), group (GRP) or other (OTHER). These appear as I, G, and O in Table 7. The Other 


\begin{tabular}{lrrrr|rrr}
\hline & $\mathrm{G}$ & $\mathrm{I}$ & $\mathrm{O}$ & & $\mathrm{P}$ & $\mathrm{R}$ & $\mathrm{F} 1$ \\
$\mathrm{G}$ & 53 & 25 & 0 & 78 & .70 & .68 & .68 \\
$\mathrm{I}$ & 9 & 90 & 1 & 100 & .66 & .90 & .76 \\
$\mathrm{O}$ & 14 & 21 & 0 & 35 & .00 & .00 & .00 \\
& 76 & 136 & 1 & 213 & .57 & .67 & .61
\end{tabular}

C-Sub1 : Multinomial Naive Bayes

\begin{tabular}{lrrrr|rrr}
\hline & $\mathrm{G}$ & $\mathrm{I}$ & $\mathrm{O}$ & & $\mathrm{P}$ & $\mathrm{R}$ & $\mathrm{F} 1$ \\
$\mathrm{G}$ & 39 & 27 & 12 & 78 & .51 & .50 & .50 \\
$\mathrm{I}$ & 21 & 70 & 9 & 100 & .63 & .70 & .66 \\
$\mathrm{O}$ & 17 & 15 & 3 & 35 & .13 & .09 & .10 \\
& 77 & 112 & 24 & 213 & .50 & .53 & .51
\end{tabular}

C-Sub2 : Decision Tree

\begin{tabular}{lrrrr|rrr}
\hline & $\mathrm{G}$ & $\mathrm{I}$ & $\mathrm{O}$ & & $\mathrm{P}$ & $\mathrm{R}$ & $\mathrm{F} 1$ \\
$\mathrm{G}$ & 48 & 25 & 5 & 78 & .61 & .62 & .61 \\
$\mathrm{I}$ & 13 & 85 & 2 & 100 & .67 & .85 & .75 \\
$\mathrm{O}$ & 18 & 17 & 0 & 35 & .00 & .00 & .00 \\
& 79 & 127 & 7 & 213 & .54 & .62 & .58
\end{tabular}

C-Sub3 : Logistic Regression

Table 7: Task C Duluth Systems

class is very sparse, and C-Sub1 and C-Sub3 did very poorly on it. However, C-Sub2 (a decision tree) had slightly more success. C-Sub1 and CSub2 rely on character ngrams, while C-Sub3 uses word unigrams, bigrams, and trigrams as features.

\section{Qualitative Review of Training Data}

Finally, we qualitatively studied some of the training data for task A and saw that there is potential for some noise in the gold standard labeling. We found various tweets labeled as offensive that seemed innocuous:

- She should ask a few native Americans what their take on this is.

- gun control! That is all these kids are asking for!

- Tbh these days i just don't like people in general i just don't connect with people these days just a annoyance..

- Dont believe the hype.

- Ouch!

- Then your gonna get bitten

- there is no need to be like That
We also found tweets labeled as not offensive despite the presence of insults and profanity.

- Ppl who say I'm not racist are racist. You Are A Racist. Repeat after me

- I'M SO FUCKING READY

- Great news! Old moonbeam Just went into a coma!

- No fucking way he said this!

- Yep Antifa are literally Hitler.

- Any updates re ending your blatant \#racism as \#Windrush \#Grenfell proves you are

The annotation guidelines from the OffensEval organizers seem relatively clear in stating that all profanity should be considered offensive, although an annotator may intuitively wish to make a more nuanced distinction. Resolving these kinds of inconsistencies seems important since the data from task A is also used for task B and C, and so there is a danger of unintended downstream impacts.

\section{Conclusion}

Offensive language can take many forms, and some words are offensive in one context but not another. As we observed, profanity was often very indicative of offensive language, but of course can be used in much more casual and friendly contexts. This quickly exposes the limits of blacklisting, since once a word is on a black-list it use will most likely always be considered offensive. Identifying targeted targeted individuals or organizations using lexical features and Machine Learning was extremely difficult, particularly given the small amounts of training data. Incorporating the use of syntactic analysis and named entity recognition seem necessary to make progress.

We also encountered the challenging impact of domain differences in identifying offensive language. Our attempt to (naively) increase the amount of available training data by combining the OffensEval and HatEval data had no impact on our results, and our feature analysis made it clear that the two corpora were different to the point of not really providing much shared information that could be leveraged. That said, we intend to explore more sophisticated approaches to transfer learning (e.g., (Karan and Šnajder, 2018; Park and Fung, 2017; Waseem et al., 2018)) since there are quite a few distinct corpora where various forms of hate speech have been annotated. 


\section{References}

Valerio Basile, Cristina Bosco, Elisabetta Fersini, Debora Nozza, Viviana Patti, Francisco Rangel, Paolo Rosso, and Manuela Sanguinetti. 2019. Semeval2019 task 5: Multilingual detection of hate speech against immigrants and women in twitter. In Proceedings of the 13th International Workshop on Semantic Evaluation (SemEval-2019).

Paula Fortuna and Sérgio Nunes. 2018. A survey on automatic detection of hate speech in text. ACM Comput. Surv., 51(4):85:1-85:30.

Tommi Gröndahl, Luca Pajola, Mika Juuti, Mauro Conti, and N Asokan. 2018. All you need is love: Evading hate speech detection. In Proceedings of the 11th ACM Workshop on Artificial Intelligence and Security, pages 2-12. ACM.

Mladen Karan and Jan Šnajder. 2018. Cross-domain detection of abusive language online. In Proceedings of the 2nd Workshop on Abusive Language Online (ALW2), pages 132-137.

Ji Ho Park and Pascale Fung. 2017. One-step and twostep classification for abusive language detection on twitter. In Proceedings of the First Workshop on Abusive Language Online, pages 41-45.

F. Pedregosa, G. Varoquaux, A. Gramfort, V. Michel, B. Thirion, O. Grisel, M. Blondel, P. Prettenhofer, R. Weiss, V. Dubourg, J. Vanderplas, A. Passos, D. Cournapeau, M. Brucher, M. Perrot, and E. Duchesnay. 2011. Scikit-learn: Machine learning in Python. Journal of Machine Learning Research, 12:2825-2830.

Anna Schmidt and Michael Wiegand. 2017. A survey on hate speech detection using natural language processing. In Proceedings of the Fifth International Workshop on Natural Language Processing for Social Media, pages 1-10, Valencia, Spain. Association for Computational Linguistics.

Zeerak Waseem, James Thorne, and Joachim Bingel. 2018. Bridging the gaps: Multi task learning for domain transfer of hate speech detection. In Online Harassment, pages 29-55. Springer.

Marcos Zampieri, Shervin Malmasi, Preslav Nakov, Sara Rosenthal, Noura Farra, and Ritesh Kumar. 2019a. Predicting the Type and Target of Offensive Posts in Social Media. In Proceedings of the Annual Conference of the North American Chapter of the Association for Computational Linguistics.

Marcos Zampieri, Shervin Malmasi, Preslav Nakov, Sara Rosenthal, Noura Farra, and Ritesh Kumar. 2019b. SemEval-2019 Task 6: Identifying and Categorizing Offensive Language in Social Media (OffensEval). In Proceedings of The 13th International Workshop on Semantic Evaluation (SemEval). 